\title{
XL. Experimental contributions towards the theory of thermo-electricity
}

\section{Mr. John Prideaux}

To cite this article: Mr. John Prideaux (1833) XL. Experimental contributions towards the theory of thermo-electricity , Philosophical Magazine Series 3, 3:15, 205-215, DOI: $10.1080 / 14786443308648160$

To link to this article: http://dx.doi.org/10.1080/14786443308648160

册 Published online: 01 Jun 2009.

Submit your article to this journal $\lceil\pi$

Џll Article views: 2

Q View related articles $₫$ 
tails of an experiment upon a larger scale, which I hope soon to have an opportunity of executing, and which will detect and measure the velocity of electricity in its passage through a metallic conductor, though the rapidity of its transmission may exceed that of light: this I have proposed to effect, by increasing, in certain proportions, 1st, the velocity of the revolving mirror; 2ndly, the length of the conducting wire; and $3 \mathrm{rdly}$, the accuracy of observing the deviation of the sparks from a vertical line. If I succeed in this point, it is obvious that we shall possess a means of directly measuring the relative conducting powers of metals, and of ascertaining numerous particulars respecting ordinary electricity which we at present have no means of determining.

Intending in the ensuing session to submit to the Royal Society all the results $I$ have obtained, in reference to $a$ nerw optical means of measuring rapid motions, minute intervals of time, and feeble intensities of light, I have hitherto refrained from publishing any incomplete statement of them; but I regret that this delay should have occasioned my experiments to be so far misunderstood, that one of the earliest which suggested itself to me, and which I have always considered to be of primary importance in the series, should be proposed elsewhere, several months afterwards, as an experiment yet to be tried, and be represented also as having entirely escaped my attention. I I remain, Dear Sir, yours, \&c.

Conduit-street, Hanover-square,

C. Wheatstone. August $2,1833$.

XL. Experimental Contributions towards the Theory of Thermo-electricity. By Mr. John Pridenux, Member of the Plymouth Institution. *

THE discovery of Professor Seebeck, that a bar of antimony, 1 or of bismuth, heated in contact with a copper or brass wire, would affect the magnetic needle, was soon extended by chemists in this country and on the Continent to all the other metals which are of ready access; and a table of the thermoelectric order of these metals was soon published by Professor Cumming, and found to differ from the voltaic order, and also from that of conduction, whether of heat or electricity.

Other experimenters presently discovered thermo-electric currents in single masses of metal, which have been traced out with curious results + .

- Communicated by the Author.

+ [Papers on the thermo-magnetism of homogeneous bodies, by $\mathrm{Mr}$. Sturgeon, will be found in Phil. Ming, and Annals, N.S. vol. x. pp. 1, 116, \&c.-EDIt.] 
M. Becquerel is, I believe, the only one who has investigated the comparative force as well as the order of arrangement of metals when acting in pairs; and having ascertained these points by a course of delicate experiments, he has been led to the inference that thermo-electricity is allied to the radiation of caloric.

The deep interest attached to a subject in which the mutual reaction of heat, electricity and magnetism is made almost tangible within a very narrow compass, led me to institute the following inquiries, with the hope of their suggesting other methods of elucidation.

I. Is thermo-electricity different from that derived from other sources: i. e. a different principle, or a different combination of principles?

II. Is it produced at the expense of caloric?

III. Is the radiation of heat, or any property dependent upon it, the proximate cause of thermo-electricity?

IV. Is the proximate cause connected with the conduction of heat?

V. Will not a hot bar brought into contact with a cold one of the same metal set electricity in motion; and if so, will the currents so produced bear any peculiar relation to those produced by pairs of different metals?

\section{Is there any, and what, difference between Thermo-electricity and that derived from other sources?}

1. The most characteristic distinction between electricity and the other imponderable fluids (if such they be,) is, its [sensibly] instantaneous transmission through considerable length of solid conductors. The very low tension of thermoelectricity renders this as close a test as any to which it can be subjected. Fifty feet of iron wire (one of the worst metallic conductors,) was cut into two lengths, through which (turned at the ends, to ensure metallic contact,) two other mercury boxes were connected with those of the magnetest * A thermo-electric pair, of antimony and bismuth, heated at the point of contact, had their feet dipped, first into the mercury boxes of the magnetest, which produced a deflection of $80^{\circ}$; and were then removed to the other pair of boxes at the. end of the wires, by which the deviation was reduced to $15^{\circ}$; and so repeatedly; the interposition of such a length of iron. wire, between the excited metals and the magnetest, withholding $\frac{4}{5}$ ths of the deviation (about $\frac{1}{2} \frac{9}{0}$ ths of the current): yet was the

"I must be excused for resuming the use of this word: "galvanometer" would be hardly applicable in this instance. 
instantaneous movement of the needle as evident in one case as in the other. So far, then, as promptitude of transmission through long wires is a distinction, thermo-electricity does not differ from the other kinds.

The magnetest employed in these experiments consists of a pair of sewing needles, 3 inches long, the lower one having just enough of predominance to give them terrestrial direction. The conductor, which passes only once between them, and returns beneath the lower needle, consists of four lengths of bright copper bell-wire laid together, not twisted, the ends amalgamated, and working into mercury boxes. A pair, of copper and tinned iron, by the heat of the fingers gives a deflection about $25^{\circ}$.

A guide-cross lies between the mercury boxes, to keep always before the eyes the relations between the current and the needle.

It is constructed of two slips of card, fastened in the centre, one over the other, with sealing-wax; one representing the magnet, the other the conducting wire. On the latter an arrow is drawn (on both sides) to indicate the direction of the current; on the former a line, also on both sides, to distinguish the marked pole.

This cross turned over, on either of the slips, as an axis, or placed horizontally, vertically, or in any degree of obliquity, always exhibits the relation between the current and the needle: and being laid down, with its magnet corresponding with the position of the needle, it shows in what direction the current is passing over it; or turning it over upon the magnet as an axis, the reverse current is seen returning under it.

2. If there be any modification of electricity answering to that of Dr. Hare, a compound of electricity and caloric, this, from the mode of its generation, should be the one. But the same pair, of antimony and bismuth (1.), heated until they would drive the needle nearly round the circle, by the first impression, had not any appreciable effect on Mr. Harris's thermotest, though tried upon wires of various dimensions and different conducting powers. Thermo-electricity, then, seems to be no way distinguished as containing caloric.

Attractions and repulsions, or chemical decompositions would be precluded by its low tension; and these are its only apparent distinctions from the voltaic current, or from the electricity of the machine. 


\section{Is Thermo-electricity produced at the expense of Caloric?}

3. Into the smaller neck of a tubulated receiver was fixed an open glass tube, reaching obliquely to the bottom, and bent, siphonwise, on the outside, so as to descend a little lower than the inner end : it was then again bent upward, nearly at a right angle, and to this rising end a scale was attached.

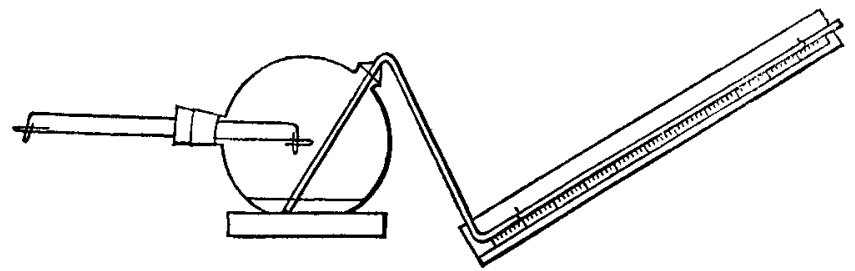

Into the receiver was poured water tinged with cochineal, and covered with a stratum of oil, to prevent evaporation by the heat. Suction being now applied to the end, the liquid remained, on the principle of the siphon, a few degrees up on the scale. A cork thoroughly soaked in grease being fitted to the open neck, the whole constituted a very sensitive air thermometer.

The cork was now perforated by two wires; one of copper, the other of tinned iron; the latter bent off at each end, so that it could be brought into contact with, or separated from the copper by a slight turn with the fingers. Both ends of each wire were doubled, flattened and polished to ensure good contact; they projected about three inches into the receiver, and nine inches outside.

Being now set in contact, the cork was drawn out, and the ends of the wires which entered the bulb were placed between a fold of sheet copper, and heated by holding them for a given time $\left(25^{5}\right)$ over an Argand gas light, being turned over at $15^{\mathrm{s}}$, to equalize the heat in both wires. The sheet copper being now drawn off, the hot ends were thrust into the bulb, and the cork pressed in air-tight. The fluid rose quickly to about $90^{\circ}$ (more or less, depending on slight differences in the heat, and in the quickness and force with which the cork was pressed in). After a few seconds, the liquid began again to descend, by the cooling of the wires. They were then separated, when the descent of the fluid was suddenly checked: on renewing the contact, it fell with renewed impetus; and so stopped and resumed its fall at every separation and renewal of the contact.

Other wires of the same and different metals were tried, and with many different corks; but the effect was always con- 
sistent, and indicated that the thermo-electric current did consume caloric in its production.

4. If this inference were true, it should follow that the wires heated to the same degree, should cool much more rapidly when kept in contact the whole time of cooling, than when kept all the time separate. They were therefore heated as before, and being thrust in, the fluid was allowed after rising to recede to $70^{\circ}$; from which its time of descent to $20^{\circ}$, i. e. $50^{\circ}$, was observed by a seconds watch, - the wires remaining in contact.

The same proceeding was repeated, the wires remaining separate.

Precision, for reasons above given (3.), was unattainable; and the conclusion was only to be drawn from the average of many experiments. This average gave $60^{5}$ for the closed wires, and less than $60^{\text {s }}$ for the separated ones; thus the cooling seemed retarded, rather than accelerated, by the contact, and consequent electrical current. All that repetition and examination could do to avoid discrepancy was attended to, and yet these experiments seemed to contradict the preceding ones.

5. The mode of interrogation was reversed. If caloric disappeared in the production of thermo-electricity, it was not unlikely that where caloric became latent, thermo-electricity might appear.

A glass siphon being suspended by the ends over a spirit lamp, tin was dropped in and melted there, until the siphon was nearly full; the ends were then connected with the poles of the magnetest by long and similar copper wires, tinned at the points. Although copper is (generally) positive to tin, no current ensued, because both ends were alike in contact with copper. A hot iron wire being now plunged into the tin, at one end of the siphon, gave a deflection of $6^{\circ}$; and when transferred to the other end, a deviation to the same amount, in the contrary direction; and so repeatedly (37.): thus manifesting a current when electricity was developed. A slip of tin was now plunged into one end: of course no deviation could be expected (37.). This tin was allowed to melt there, by which caloric must have become latent. The fusion was gradual, and in full contact with metal on all sides; the electricity, if any were produced, must therefore have been continuous, and could not have acquired tension. It would

- Not degrees of Fahreriheit, or of any other standard scale; for the heat of the air about the wires must have been very different from that about the glass.

Third Scrics. Vol. 3. No. 15. Sept. 1833. 
also have taken the copper, in preference to the tin, and the needle would have deviated accordingly.

But no deviation occurred.

Mercury, which is pretty near tin in thermo-electric power, was then substituted; and being heated to the melting point of tin, a warm iron wire was plunged in, which gave a deviation of $5^{\circ}$ : but iron not coming well into contact with mercury, a tinned iron wire was tried, by which a deviation of $6^{\circ}$ was obtained as before (5.). In this slips of tin were successively melted; but without any manifestation whatever on the needle.

Here we have no evidence of thermo-electricity, on the disappearance of caloric; in the preceding experiments we had no evidence of the disappearance or abstraction of caloric, in the production of thermo-electricity. To what, then, are we to attribute the contrary evidence of the previous experiments, as the apparatus was certainly air-tight?

6. When the wires were separated, the whole heated surface was exposed to the air in the bulb; and the portion between the heated ends would be most expanded, and least affected by the cooling influence of the glass. When they were in contact, this, the most effective part of the heated surface, was covered up. Hence a momentary expansion upon opening, and contraction upon closing the contact of the wires.

But since when thus in contact they cool less quickly, in proportion as they have less effect upon the air; so the whole time of descent, for $50^{\circ}$ of the scale, should be greater, rather than less, when closed than when open.

If this were the true explanation, homogeneous wires should answer as well as those of different metals. Accordingly, two copper wires were passed through a cork, bent and heated as before (3.); and the descent of the fluid was found to be suddenly stopped and resumed, at the instants of opening and closing the contact, just as when the wires were of two different metals.

7. The evidence is therefore opposed to the expenditure of caloric in the production of thermo-electricity, and to the converse. Yet I thought the opposing experiments worth stating, (as they were not unlikely to occur to others, to show that they had been tried, and shown to be inconclusive.

1II. Horw far is the Radiation of Heat, or any collateral property, concerned in the Development of Thermo-electricity?

8. The result of $M$. Becquerel's exact researches, above quoted from the Ann. de Chim. et de Phys., Aug. 1829, is, that the only known property of heat in which the metals take 
the same order as in thermo-electricity, is that of radiation; and therefore he supposes that two pieces of hot metal, in contact, radiate in the same proportions as they would if separate and exposed; and that thermo-electricity is a result of this inter-radiation.

This is certainly applying to the term radiation a new meaning, very little analogous to its ordinary acceptation*. Yet the same quality, whatever it may be, which occasions radiation to be more or less copious, may also determine a superficial communication, proportionately copious, though different in mode. We have evidence, too, that the superficial absorbent power for heat, is proportionate to the radiating power; and it seemed not very improbable that here might be found some trace towards the solution of the problem.

9. If the effects were due to any cause connected with, or related to radiation, they should be modified by alterations in the surfaces; and by making the surfaces of a thermo-electric pair identical, their reaction should be greatly weakened, if not neutralized altogether.

An iron and a copper wire, of the same size $\left(10 \times \frac{1}{16}\right.$ inch $)$, were well tinned from end to end, and twisted together for an inch. A similar pair, untinned, but polished, were similarly joined; and the feet tinned for $\frac{1}{2}$ an inch, that the contacts with the mercury might be alike. Instead of the tinned wires having their efficacy impaired, they gave greater deflections than the clear ones. This unexpected fact, verified in a great number of ways, led to a long course of experiments; the general bearings of which will occupy a subsequent section (VIII.).

I am at a loss to reconcile with this fact any cause connected with radiation.

10. To try radiation more directly, and on a larger scale, a plate of tinned iron was wired round with tinned iron wire, which projected at one corner, for a conductor. A sheet of soft tin was also wired round with copper wire, projecting in the same manner, and for the same purpose. The sheet tin being laid on the tin plate, with a leaf of bank post paper between, to prevent contact, the conductors were plunged into the mercury of the magnetest, and a couple of lamps placed under the tin plate. The whole soon became hot, without deflecting the needle. The tin sheet was now lifted off, the paper removed, and a few threads spread about the

- [It appears to us, on the contrary, that $M$. Becquerel here uses the term radiation in a sense perfectly similar to its ordinary acceptation : see Sir J. Herschel's remarks on the process by which radiation and conduction slide into each other, in his Prel. Disc. on Nat. Phil.-EDit. ] 
tin plate, to keep the sheet tin from touching it, whilst their surfaces should be fully exposed for radiation, at an extremely small distance. No deflection ensued on thus replacing the sheet tin; but on putting a slip of tin-foil in contact with both, the needle started off $25^{\circ}$.

A plate of polished copper was similarly used, first with sheet tin, afterwards with the tin plate; but with no effect whatever, in either case, until metallic contact was made.

The total inefficacy of such large radiating surfaces, added to the result of the preceding experiments, discourages the hope of tracing the canse of thermo-electricity to any superficial action analogous to radiation; and more convincing evidence to the same effect will appear (20. et seq.).

In fact $I$ have been unable to find any table of radiation corresponding with Becquerel's thermo-electric order.

\section{Is the proximate cause of Thermo-electricity in any way connected with Conduction?}

12. Having previously found reason, from the experiments of others as well as my own, to conclude that the heat produced by electricity in metallic conductors (and, with dne allowance, in liquids and air,) is in the order of, and probably in proportion to the resistance it encounters in the body heated, whether that resistance was owing to inferior conducting property or diminished thickness, I was led by the following experiment, compared with some of Becquerel's, to suspect a reciprocity of action, such that electricity, restricted in its passage, producing heat; so heat, allowed to flow more freely, might produce electricity.

The circuit from a voltaic coil was divided into 5 parts, the two ends next the coil, and the 5th or middle piece, being of stout copper wire, the ends each 2 feet, the mirddle piece 4 feet long; the connexions from each end to the middle piece were fine silver wire $\frac{1}{10} 0$ th of an inch thick. Thus no electricity, positive or negative, could reach the middle piece except through these fine wires.

The charge being now made such as to warm the silver wires, a magnetic needle was moved along the circuit, which was laid (ap ortion of the middle piece, of course, excepted,) in the magnetic mericlian. Its equidistance from the conducting wire was ensured, by the card, on which it was supported, being kept always in contact therewith. When over the copper, either of the middle or end pieces, the divergence was $36^{\circ}$; when over either of the silver wires, $26^{\circ}$. Thus heat seemed to be produced, by restriction of the current, on entering the 
silver wire; and the full electrical effect appeared to be restored when the obstruction was overpassed.

13. Becquerel found (ubi supra) that a circle of platinum wire, of equal diameter throughout, produced no current when heated in any part; but a knob being made in the circle, and heat applied near it, on either side, a current set always from the heated point towards the knob, where the heat had room to diffuse itself.

Also, that on soldering together the ends of his magnetest wire, no current ensued, when the point of junction was heated even to redness; but on touching the wire on either side of the heated point with a cold piece of the same metal, the current immediately set towards the part so touched.

14. It is true that the tables of conduction for heat and electricity, contrasted by Cumming with that of thermo-electricity, deny the presumption of any connexion between them. But there would appear to be two properties of conduction for caloric,-promptitude and final efficacy. For although we handle platinum wire, at two inches distance from a red-hot point, in operations with the blowpipe, which hardly any other metal will admit of, yet M. Despretz found that a rod of platinum eventually became hotter, at a given distance from a given source of heat, than any other metallic rod of equal dimensions; and if thermo-electricity be influenced by conduction at all, it must be by promptitude of conduction, for very short distances: whilst the tables hitherto published refer to a property compounded of the two, or intermediate between them.

A copper and an iron wire, drawn so that equal lengths corresponded to the atomic weights, were bound with thread for an inch each, cut off at $\frac{1}{2}$ an inch above the binding,-this $\frac{1}{2}$-inch bent over in the form of a hook, and the end filed flat. The two wires, thus prepared exactly alike, were fixed to a little wire frame, to keep them parallel and separate, and the filed ends dipped into melted lard. A couple of orange peas, of equal weight and size, were also dipped in the lard. When cold, the cohesion of the lard was sufficient to support the little balls against the ends of the wires. The bound part of the wires was now plunged into warm mercury, and the ball soon fell from the copper by fusion of the lard. The wires were then cooled by plunging the bound part into cold mercury, and the balls interchanged. On replunging into warm mercury, the ball fell again from the copper; and this always took place on repeated interchange of the balls, and alternation of cold and heat; the ball falling every time from the copper, until the mercury was cooled so far that the lard would not melt, and so that neither ball would fall. This was decisive 


\section{Mr. Prideaux on the Theory of Thermo-electricity.}

in favour of the conducting power of copper, down to a very low heat, and at $\frac{1}{2}$ an inch distance.

15. To try at still shorter distances, two other lengths of the same wire were twisted together for $\frac{1}{2}$ an inch, then separated for an inch, and the other ends brought near together, for holding in a pair of cold pliers. (The twist was tarnished, to prevent metallic contact between the mercury and copper, which also was the object of the thread-binding in the former part of the experiment.) Spots of lard were now placed, at equal distances from the twist, on the iron and on the copper, and the twist plunged into the warm mercury. The lard became transparent (by melting) first on the copper: and this on repetition, whatever the distances, down to $\frac{1}{8}$ th of an inch; and whatever the temperature, so long as it was sufficient to melt the lard at all. Varying the sizes and proportions of the wires, tinning their surfaces, to prevent unequal waste of heat, in transitu, by radiation, did not alter the result. In every instance, for equal distances, the lard first became transparent upon the copper wire; which is thereby proved, for low heats and short distances, to retain its character as the better conductor for caloric.

16. To ascertain the conducting powers for electricity of very low tension, a pair of iron and a pair of copper wires, atomically proportioned as before, and each 2 inches long, had $\frac{1}{2}$ an inch at each end bent down at right angles, and the points tinned, for equal contact with the mercury. Through these wires, two other mercury boxes were connected with those of the magnetest, an iron and a copper wire being employed on each side. Into the further boxes dipped the ends of a thermo-electric pair, of copper and tinned iron, of which the other ends were twisted together, and the twist was exposed to an equable heat, until the needle became stationary at $18^{\circ}$.

The short iron wires were now removed, leaving the conduction to be carried on by the short copper ones only: no appreciable change took place in the deflection.

The iron wires being now replaced, the copper ones were removed, leaving the conduction entirely to the iron. The needle receded more than a degree, and sometimes nearly $2^{\circ}$, confirming the superiority of copper as an electrical conductor, for extreme low tension and very short distances.

17. But as heat also enters into the question, the apparatus remaining as described, a spirit-lamp was brought under the middle of the copper leg of the thermo-electric pair, and continued there until the point acted upon by the flame became red hot. Little or no change took place in the deflection. 
The lamp being then removed to the iron leg, the needle receded $2^{\circ}$ before the beat had attained redness. Thus the conducting power of iron suffered more from heat than that of copper.

18. Iron being thus proved to be inferior in conducting power, both for heat and electricity, in circumstances directly in point, to copper, cannot owe its thermo-electric superiority to that property; and, extending the analogy to other metals, the inference at the beginning of this section would seem to be refuted. But the following section will bring the question before us in a different point of view.

[To be continued.]

\section{Proceedings of Learned Societies.}

\section{ROYAL SOCIETY.}

April 18. A PAPER was read, entitled, "On Improvements in the

A Instruments and Methods employed in determining the Direction and Intensity of 'Terrestrial Magnetism." By Samuel Hunter Christie, Esq. M.A. F.R.S.

The tedious nature of the observations by which the direction and intensity of the terrestrial magnetic force are determined, and the uncertainty attending the results when obtained, have long been a subject of regret to all who are engaged in the investigation of the phænumena of terrestrial magnetism. Sensible of this, the author's attention has at different times been turned to the impravement of the instruments employed for these purposes; and in this communication he proposes methods by which he considers that these instruments might be so improved that the results should be obtained with greater facility and also with greater certainty. The uncertainty attending the results obtained with the dipping needle, as at present constructed, arises principally from the two sources, friction upon the axis, and the want of coincidence of the needle's centre of gravity with the axis of motion; the latter rendering recessary the inversion of its poles: The author suggests a method by which he considers that, probably, the friction may be diminished; but he has principally directed his attention to obviate the necessity of the inversion of the poles.

In order to remove the practical difficulty attending the adjustment of the centre of gravity to the asis of motion, an operation in which the artist rarely, if ever, completely succeeds, the author proposes to dispense with this condition; and shows how the dip may then be determined, without the necessity of inverting the poles of the needle, the position of its centre of gravity having been determined previously to its being magnetized. The advantages attending the method proposed by the author are not, however, restricted to the determination of the dip with greater accuracy and greater facility: a further and still greater advantage attending the use of a dipping needle on the principle he proposes, is, that a measure of the terrestrial magnetic. intensity will be obtained by the same observations which give the 